\title{
The role of incentive learning in instrumental outcome revaluation by sensory-specific satiety
}

\author{
BERNARD W. BALLEINE \\ University of California, Los Angeles, Califormia \\ and \\ ANTHONY DICKINSON \\ University of Cambridge, Cambridge, England
}

\begin{abstract}
Hungry rats were trained to perform two instrumental actions, one for salt- and the other for lemonflavored polycose solution. When they were sated on one of these two outcomes by prefeeding immediately prior to a choice extinction test, the action trained with the prefed solution was performed less than the other action. The subsequent experiments examined the role of incentive learning in this specific satiety-induced outcome revaluation effect. The second experiment demonstrated that the experience of consuming a flavored polycose solution to satiety enabled the state induced by polycose consumption to control the devaluation of the flavored outcome. By contrast, the third study found that, although devaluing the prefed outcome, specific-satiety treatments could induce a relative inflation in the incentive value of other food outcomes. The final two studies demonstrated an increased outcomedevaluation effect in instrumental performance when these devaluation and revaluation effects were combined. Taken together, these studies demonstrate that specific satiety treatments produce changes in outcome value that depend upon incentive learning.
\end{abstract}

In recent years, the outcome-devaluation procedure has been used to great effect in exploring the psychological determinants of instrumental action in rats (Adams \& Dickinson, 1981; Balleine 1992; Colwill \& Rescorla, $1985)$. In this procedure, rats are initially trained to perform an instrumental action for access to a food or fluid before this outcome is devalued. The animal's propensity to perform the instrumental action is then assessed in an extinction test. The rationale for this procedure is straightforward: if the performance of the instrumental action reflects encoding of the action-outcome relation acquired during training, then subsequently devaluing the outcome should reduce performance on test, a prediction that has been consistently upheld (see Colwill \& Rescorla, 1986, and Dickinson \& Balleine, 1994, for reviews).

The potency of this procedure is nowhere better demonstrated than in Rescorla's (1990, Experiment 1) finding that, in instrumental conditioning, even motivationally incidental features of the instrumental outcome are encoded. In that experiment, thirsty rats were trained to

The research reported in this article was supported by grants from the Academic Senate, UCLA, the National Institute of Mental Health, NIMH Grant MH 56446, and the European Commission BIOMED 2 program. The authors thank Claire Garner, Suzette Glasner, Susan Sorenson, and Michaeline Hanney for their assistance in running the experiments. Requests for reprints and correspondence concerning this article should be addressed to B. W. Balleine, Department of Psychology, University of California, Los Angeles, 405 Hilgard Avenue, Los Angeles, CA 90095-1563 (e-mail: balleine@psych.ucla.edu).

Accepted by previous editor, Robert A. Rescorla leverpress and to chainpull, with both actions earning water. For one action, however, the water was made sour through the use of a small quantity of hydrochloric acid; the other action earned water made bitter through the use of a small quantity of quinine. An aversion was then conditioned to either the bitter or sour water by pairing it with lithium chloride, after which an extinction test was conducted on the levers and chains. In spite of the fact that the critical motivational property, that is, the hydrating property, was the same for both of the instrumental outcomes, Rescorla found that animals performed fewer of the actions that, in training, had delivered the poisoned outcome, indicating that they had encoded the incidental sour and bitter taste features of the water outcomes as consequences of their instrumental actions.

Rescorla's (1990) demonstration is important, because it shows that the incentive value of an outcome can be mediated by a motivationally arbitrary feature, its taste. If this devaluation treatment modified the degree of thirst or the animal's encoding of the motivationally relevant properties of fluid outcomes, the performance of both of the actions should have been reduced on test and to a similar degree. As such, this finding provides evidence for a highly specific encoding of the instrumental outcome, one that allows for modification of the incentive value of a taste feature while leaving the value of features common to the other fluid outcome (e.g., temperature, texture, appearance) relatively unaffected.

Evidence that, in instrumental conditioning, animals can encode the outcome of their actions is not confined to devaluation effects accomplished using taste-aversion con- 
ditioning. One of the simplest means available for demonstrating this effect is the devaluation of a food outcome by a specific satiety treatment. In this procedure, the food outcome is devalued by giving rats extensive access to it until it is no longer consumed, a treatment that appears to devalue selectively the prefed outcome. In one example, Colwill and Rescorla (1985) trained hungry rats to perform two actions, leverpressing and chainpulling, with one action earning access to a sucrose solution and the other to food pellets. After this training, the animals were placed on a free-feeding schedule and then allowed to consume one of these outcomes to satiety immediately before they were given a choice test between the lever and chain in extinction. Colwill and Rescorla found that, on test, animals performed fewer of the action that, in training, had delivered the outcome on which they were sated prior to the test.

Although this simple procedure produces replicable devaluation effects (e.g., Dickinson, Campos, Varga, \& Balleine, 1996), little is known about how the specific satiety treatment devalues the instrumental outcome. As the outcomes employed by Colwill and Rescorla differed not only in their taste but also in their tactile features (solid vs. fluid) and nutritional composition, it is not clear whether the devaluation produced by the satiety, like that induced by aversion conditioning, can be mediated solely by taste. Experiment 1 addressed this issue by replicating the Colwill and Rescorla procedure but using outcomes that differed solely in their taste.

\section{EXPERIMENT 1}

Animals were first trained to press two levers on a concurrent schedule, with performance on both of the levers delivering a $20 \%$ polycose solution. Performance on one lever, however, delivered salty-tasting polycose, while pressing the other lever delivered sour-tasting polycose. After this training was complete, one of the two polycose solutions was devalued using a specific-satiety procedure. This was accomplished by giving the animals access to one of the two solutions for $1 \mathrm{~h}$ immediately prior to a choice test conducted on the two levers in extinction. If the specific-satiety treatment can act to reduce selectively the incentive value of the taste feature of the instrumental outcome on which the animals were sated, the animals should be predicted to perform fewer presses on the lever that, in training, had delivered the polycose solution on which they were sated prior to the test.

\section{Method}

Subjects and Apparatus. The subjects were 16 naive adult female Long-Evans rats that were housed singly. Training and testıng took place in eight Med Associates (East Faırfield, VT) operant chambers housed in sound- and light-resistant shells. Each chamber was equipped with two pumps, each fitted with a synnge which, when operated, delivered $0.1 \mathrm{ml}$ of a $20 \%$ polycose solution into a recessed magazine positioned in the center of the front wall One syringe held polycose dissolved in a $0.1 \mathrm{M}$ sodium chloride solution; the other held polycose dissolved in tap water to which $3^{\circ} 0$ lemon juice was added. Each chamber also contanned two re- tractable levers that could be inserted to the right and left of the magazine and was illuminated by a $3-\mathrm{W}, 24-\mathrm{V}$ houselight mounted on the top-center of the wall opposite the magazine. A microcomputer equipped with the MED-PC program for on-line control (Med Associates) controlled the equipment and recorded the leverpresses. For the specific-satiety prefeeding, one of the polycose solutions was placed in a calibrated glass drinking tube with a metal spout. This tube, which, when presented, always held $50 \mathrm{ml}$ of solution, could be fastened to the front of the animal's home cage.

Procedure. The rats were maintained on a 23 -h food deprivation schedule throughout instrumental training, with free access to tap water in their home cages by being given 1-h access to their maintenance diet each day. Each session started with the onset of the houselight and, when appropriate, the insertion of the levers; it ended with the offset of the lights and retraction of the levers. Initially, all subjects received two sessions of magazine training, in each of which 15 presentations of each of the two polycose solutions were given on a random time (RT) 60 -sec schedule with the levers withdrawn. In the next session, the animals were trained to press one of the levers and then, in the subsequent session, to press the other lever, with half of the animals initially trained on the left lever and the other half on the right lever. Each leverpress was reinforced during both sessions until 30 outcomes had been earned. For 8 of the rats, pressing the left lever delivered the salt polycose and the right lever delivered the sour polycose. For the remaining rats, these action-outcome assignments were reversed. During concurrent training with both levers present, the two polycose solutions became available on a single random interval (RI) schedule, with the available outcome specified as either the salt or sour polycose with equal probability. The subroutıne controlling the schedule was suspended whenever an outcome became available and was restarted only once it had been delivered. During this training, a total of 30 outcomes were delivered in each session, 15 contingent on one action and 15 on the other. As soon as anımals had obtained 15 of one type of outcome, this type was no longer delivered and the schedule immediately programmed the interval to the other outcome. The session continued until 15 of the other outcome had been delivered, at which point it ended. This type of concurrent schedule ensures that, on average, the outcomes are evenly distributed to the two actions across the session whatever the relative distribution of performance of the two actions. The nominal RI schedule parameter was 5 and $10 \mathrm{sec}$ for the first two sessions of concurrent training and $15 \mathrm{sec}$ for the next five sessions.

On the day following the final training session, all of the rats were given the opportunity to consume one of the polycose solutions for $1 \mathrm{~h}$. For half of the animals in each action-outcome assignment condition, the prefed outcome was the salt polycose; for the remainder, it was sour polycose. Immediately following this prefeeding, the animals were placed in the operant chambers and the rate of pressing on the two levers was assessed in a single 10 -min choice extinction test. No outcomes were delivered during this session.

Throughout this series of experiments, an alpha level of .05 was used to assess statıstical significance.

\section{Results and Discussion}

The results from the extinction test are presented in Figure 1 separately for the two actions and for each 2-min period of the extinction test. As is clear from this figure, the animals consistently performed fewer presses on the lever that, in training, had delivered the subsequently prefed or devalued polycose solution than they did on the other lever which had been trained with the nonprefed or valued outcome, a description that was supported by the statistical analysis. A two-way analysis of variance was conducted, with within-subjects factors of devaluation, 


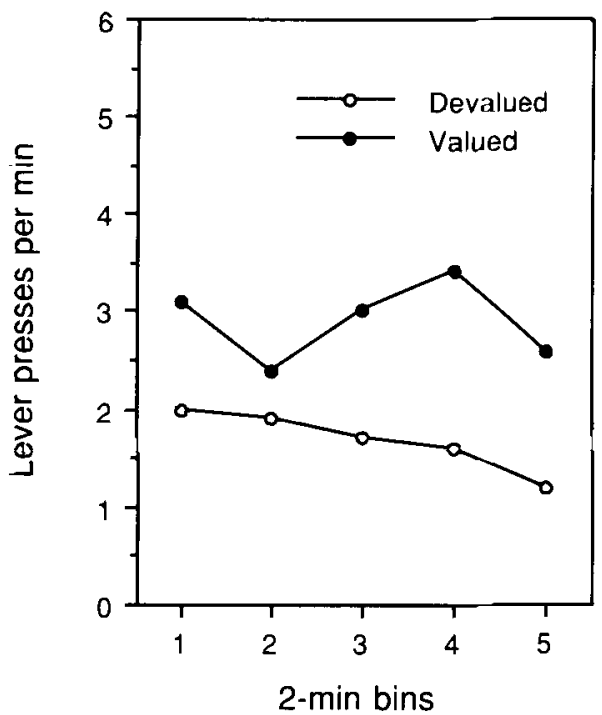

Figure 1. Experiment 1: Mean leverpresses per minute during the choice extinction test. The results are presented separately for leverpresses that, in training, delivered the outcome subsequently devalued by prefeeding prior to the test and for the action that, in training, delivered the outcome that remained valued.

which separated performance on the devalued and valued actions, and of bins, which distinguished performance on the two actions into 2-min bins during the extinction session. This analysis revealed a significant effect of devaluation $[F(1,15)=6.75]$ but neither an effect of bins $[F(9,135)=1.2]$ nor an interaction between these factors $[F(9,135)=1.3]$.

This evidence of outcome devaluation emerged on test; there was no difference in the performance of the two actions during training prior to the devaluation treatment. Comparable analysis conducted on performance in the final training session revealed no effect of devaluation $(F<1)$. In the final training session, the rates of leverpressing for the to-be-devalued and to-be-valued outcomes were 6.4 and $6.2 \mathrm{presses} / \mathrm{min}$, respectively.

This result replicates previous demonstrations that an instrumental outcome can be devalued by prefeeding to satiety (e.g., Colwill \& Rescorla, 1985; Dickinson et al., 1996). Moreover, by employing outcomes that differed only in their taste, the present study shows that the incentive value of an instrumental outcome can be carried by its taste alone, a conclusion that accords with Rescorla's (1990) demonstration of taste-mediated outcome devaluation by aversion conditioning. Nevertheless, how the specific-satiety treatment acts to reduce the incentive value of the instrumental outcome remains unclear.

Balleine and Dickinson (1991) suggested that outcome devaluation by taste-aversion conditioning was mediated, at least in part, by a process that they referred to as incentive learning (see Dickinson \& Balleine, 1994, for a review). They trained thirsty rats to leverpress and chainpull in a single session, with one action earning salty water and the other action earning sweetened water on a con- current schedule of reinforcement. After this session, rats were given an injection of lithium chloride in the hope of conditioning an aversion to both the salt and the sweet tastes. Prior to an extinction test with the levers and chains, the rats were given the opportunity to drink a small quantity of either the salted or the sweetened water, a treatment that had the effect on test of reducing the animals' performance of the action that, during training, had delivered the reexposed outcome relative to the other action. As with Rescorla's (1990) experiment, this finding indicates that incentive value can be mediated by the specific taste features of the instrumental outcome. More importantly, it demonstrates that outcome devaluation is, at least in part, a consequence of the animal's experience of a change in the attractiveness or palatability of the taste features of the outcome during the reexposure which, in turn, leads to the assignment of a lower incentive value to the outcome. The purpose of the next study was to determine whether such incentive learning also played a role in outcome devaluation induced by specific satiety.

\section{EXPERIMENT 2}

Balleine (1992) suggested that outcome devaluation by specific satiety might reflect a special case of devaluation by a shift in primary motivation, a devaluation treatment that has also been shown to involve a form of incentive learning. This suggestion is based on the observation that, in the case of some free-operant procedures (see Balleine, Garner, Gonzalez, \& Dickinson, 1995), instrumental performance for food outcomes is affected by a reduction in food deprivation only when animals have had prior experience of the outcome in the reduced deprivation state (Balleine, 1992). For example, having trained hungry rats to press a lever for a food outcome, Balleine (1992) failed to detect any effect of the level of food deprivation in a subsequent extinction test; animais with free access to their maintenance diet pressed just as much as rats that had been deprived of food in their home cages prior to this test. For a reduction in the level of food deprivation between training and testing to influence test performance, prior experience with the instrumental outcome in the undeprived state was required. When animals were exposed to the instrumental outcome in the undeprived state either before instrumental training (Balleine, 1992) or between training and testing (Balleine \& Dickinson, 1994), the deprivation level on test then acted to control performance and the rate of performance was reduced in an extinction test.

We (Balleine, 1992; Dickinson \& Balleine, 1994) have interpreted this and related findings as evidence that primary motivational states, such as the states of hunger and of satiety, do not always exert direct control over instrumental actions trained with relevant outcomes. Rather, animals have to learn about the incentive value of an outcome in a particular motivational state through consummatory experience with the outcome when they are in that state. Given this analysis of motivational control in 
general, Balleine (1992) suggested that, in the specific satiety treatment, prefeeding the animals with the instrumental outcome might allow them, through the progressive induction of satiety, to learn about the relatively low incentive value of the outcome in this state, an effect that is manifest in reduced instrumental performance in the test that immediately follows the prefeeding.

Two lines of evidence support this incentive learning account of outcome devaluation by specific satiety. First, Balleine (1992, Experiment 4) compared the effect of prefeeding with the outcome to that produced by manipulating the home-cage deprivation state. Once again, the rats were trained to leverpress for food pellets while food deprived before they were tested in the undeprived state. In the standard incentive learning condition, undeprived rats were exposed to a limited number of the food pellets prior to any instrumental training, whereas a second group was simply prefed the pellets to satiety immediately prior to the instrumental test. Significantly, both of these groups showed a comparable level of performance on test, which was markedly lower than that of animals that had not been previously exposed to the outcome in an undeprived state. Thus, standard incentive learning and prefeeding procedures produced a comparable devaluation effect.

The second line of evidence concerns the outcome specificity of incentive learning. In Colwill and Rescorla's (1985) original study, devaluation of an instrumental outcome by prefeeding was, at least in part, specific to the prefed outcome. There are now numerous demonstrations that incentive learning shows comparable specificity. In one study (Balleine \& Dickinson, 1994, Experiment 2), for example, food-deprived rats were trained to perform two actions, leverpressing and chainpulling, on a concurrent schedule, with one action earning access to food pellets and the other to a maltodextrin solution. Following training, the rats were given a number of brief reexposure sessions in which they were allowed to consume one of the instrumental outcomes in the training statethat is, food deprived-and the other in the undeprived state on alternate days. On the next day the rats were given a single choice test between the chain and the lever when undeprived, with the test conducted in extinction. Congruent with the specific-satiety effect observed by Colwill and Rescorla (1985), Balleine and Dickinson (1994) found that animals performed significantly fewer of the actions that, in training, had delivered the outcome to which they were subsequently reexposed in the undeprived state. The similarities between the two procedures for devaluing an outcome establish a case for arguing that incentive learning processes play a role in outcome devaluation by specific satiety.

According to an incentive learning account, the devaluation effect observed in Experıment 1 was a consequence of learning that, when in the state of satiety induced by prefeeding on a flavored polycose solution, the prefed polycose outcome has a low incentive value. In the next study, we evaluated this account by separating the opportunity for incentive learning from the prefeeding. In this experiment, rats were initially trained concurrently to press two levers with one delivering salt polycose and the other sour polycose, as in Experiment 1. On each of the 2 days that followed the completion of instrumental training, the rats were exposed to one of the polycose outcomes used in training for $1 \mathrm{~h}$, either the salt or the sour polycose, using the specific-satiety devaluation treatment, again as in Experiment 1. Finally, all rats were given two brief choice extinction tests on the levers on the 3rd and 4th days after the completion of training, in counterbalanced order, to assess whether the polycose-induced satiety state controlled the incentive value of the taste presented with the polycose during the exposure phase. To achieve this, immediately prior to one test, the animals were allowed $1 \mathrm{~h}$ of free access to an unadulterated $20 \%$ polycose solution, that is, polycose containing neither the salt nor the sour tastes. For the other test, the animals were food deprived as in training.

According to the incentive learning account, the exposure phase should have allowed the rats to learn about the reduced value of the taste consumed when in the state of satiety induced by extended consumption of the accompanying polycose. As a consequence, this account predicts that the rats should perform fewer presses on the lever trained with this outcome in the test conducted after extended exposure to the unadulterated polycose solution. In addition, if, as suggested, any devaluation effect is controlled by the polycose prefeeding, this account should also predict that such an effect should not emerge in the test conducted when the animals are not prefed, that is, in the absence of the state on which the reduced value of the taste presented in the exposure phase should have become conditional.

\section{Method}

Subjects and Apparatus. Sixteen naive adult female LongEvans rats were housed and maintained under the conditions and trained in the apparatus used in Experiment 1

Procedure. The procedure for instrumental training was exactly as described in Experiment 1. The rats were maintained on a 23-h food deprivation schedule throughout instrumental training. After two sessions of magazıne trainıng, all animals were first trained to press one lever and then, in the subsequent session, to press the other lever. For 8 of the subjects, pressing the left lever delivered the salt polycose and pressing the right lever delivered the sour polycose; the remaining 8 subjects received the opposite action-outcome assignment. During concurrent training on both levers, the polycose outcomes became avallable on a single RI schedule as described in Experiment 1 . The nominal RI schedule value was again 5 and $10 \mathrm{sec}$ for the first two sessions of concurrent training and $15 \mathrm{sec}$ for the next five sessions.

In the next phase, all animals experienced the specific-satiety devaluation treatment with one of the outcomes. On the day after the final training session, each animal was given the opportunity to consume one of the instrumental outcomes from the drinking tubes in their home cages for $1 \mathrm{~h}$. For half of the anımals in each actionoutcome assignment condition, the outcome to which they were exposed was $50 \mathrm{ml}$ of the salt polycose; for the remainder, it was $50 \mathrm{ml}$ of the sour polycose. At least $2 \mathrm{~h}$ after this session, all animals were allowed access to their maintenance diet for $30 \mathrm{~min}$. This procedure was then repeated on the next day, thus giving all anımals two opportunities to consume one of the outcomes to satiety.

On the following day, half of the animals in each action outcome and exposure condition were allowed to consume an unadulterated 
$20 \%$ polycose solution from the drinkıng tubes for $1 \mathrm{~h}$. The remainder received exposure to drinking bottles, but these contained no solution. Immediately after this period, the animals were placed in the operant chambers and performance on the two levers was assessed in a 10-min choice extınction test. No outcomes were delivered during this session. The following day, the rats given the empty-bottle test the day before were allowed to consume the unadulterated polycose, whereas those exposed to the polycose were now exposed to the empty bottle for $1 \mathrm{~h}$ prior to a second extınction test conducted exactly as described for the first test.

\section{Results and Discussion}

Figure 2 shows the rate of performance on each action in 2-min bins throughout the two tests, with performance in the test preceded by consumption of polycose presented in the left panel (satiety test) and performance in the test preceded by exposure to the empty bottle shown in the right panel (hunger test). A devaluation effect was observed in the satiety test in that the rats performed fewer presses on the devalued lever, which in training had delivered the polycose solution to which they were exposed in the specific-satiety treatment, than they did on the other, valued, lever. This effect was not observed in the test conducted hungry, however. In addition, in comparing the two tests, it was clear that sating the animals on the polycose had a general suppressive effect on performance of both actions. Performance on both levers in the test conducted with hungry rats exceeded that in the test conducted after the rats were sated on the unadulterated polycose.
For the statistical analysis, a three-way analysis of variance was conducted with within-subjects variables of test, to distinguish performance in the satiety and hunger tests, devaluation, to distinguish performance on the devalued lever from that on the valued one, and bin, to distinguish performance in each of the 2-min periods of the tests. This analysis revealed a main effect of test $[F(1,15)=55.5]$ and of bin $[F(4,60)=34.7]$, but no main effect of devaluation $[F(1,15)=1.1]$. There was, however, a significant interaction between test and devaluation $[F(1,15)=5.9]$. Simple effects analysis conducted on this interaction revealed a significant effect of test on the performance of both the devalued $[F(1,15)=29.6]$ and the valued actions $[F(1,15)=72.7]$ and, more importantly, a reliable effect of devaluation in the satiety test $[F(1,15)=16.5]$ but not in the hunger test $(F<1)$. Again, this effect of the specific satiety treatment emerged on test and was not present in the training data. Comparable analysis of the final session of training revealed no main effects or interactions involving devaluation $(F<1)$. The rates of pressing on the to-be-devalued and nondevalued levers in that session were 6.5 and 6.4 leverpresses $/ \mathrm{min}$, respectively.

The results of Experiment 2 demonstrate that incentive learning plays a role in the devaluation of an instrumental outcome by specific satiety. Although prolonged exposure to the polycose prior to the test reduced performance of both actions to some degree, this treatment clearly induced a greater devaluation effect on the action

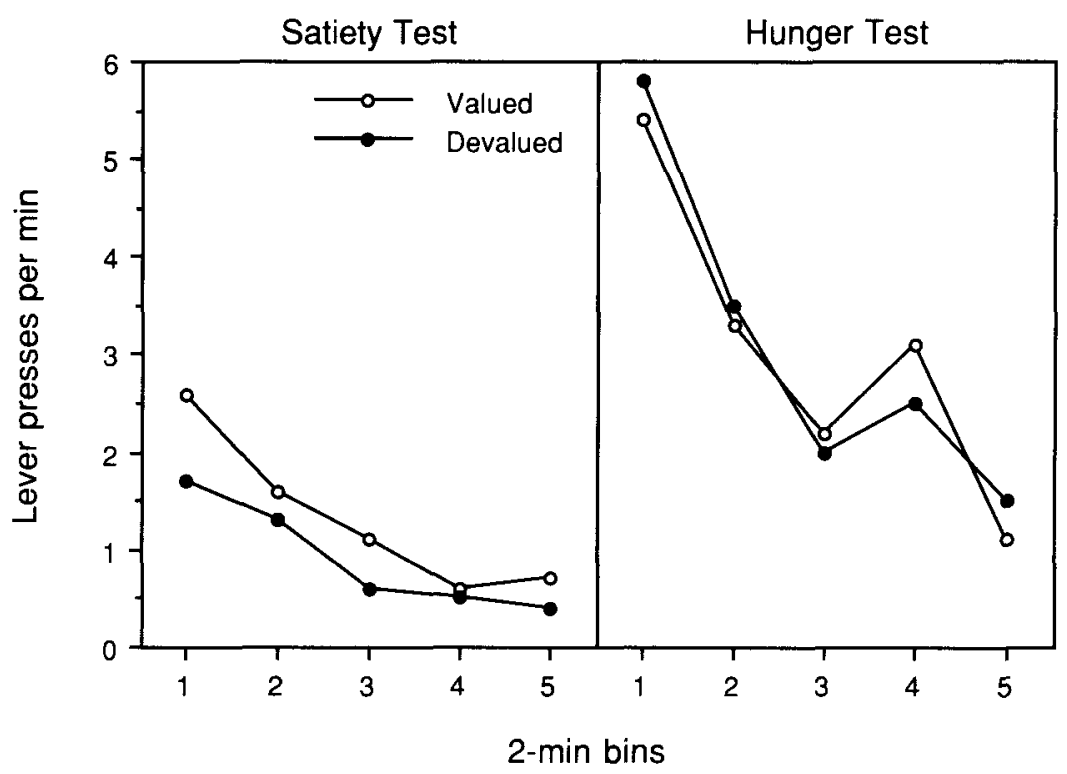

Figure 2. Experiment 2: Mean leverpresses per minute during the choice extinction tests. The results are presented separately for the two tests: the satiety test conducted after prefeeding (left panel) and the hunger test conducted in the absence of this treatment and when the rats were, therefore, hungry (right panel). The results are presented separately for leverpresses that, in training, delivered the outcome subsequently devalued by the specific-satiety treatment and for the action that, in training, delivered the outcome that remained valued. 
that was trained with the particular polycose solution on which animals were sated during the specific-satiety devaluation phase. The reduction in the incentive value of the particular taste feature (i.e., salt or sour) consumed during this phase came under the conditional control of the state of satiety induced by consumption of the polycose, and thus the effects of devaluation were observed only in the test conducted after the induction of satiety on the unadulterated polycose and were not present in the test conducted with the rats hungry. This result strongly suggests that, in the standard specific-satiety procedure, animals learn during prefeeding to assign a low incentive value to the taste of the outcome conditional upon the state induced by consuming the outcome to satiety, and this low value then controls instrumental performance during the subsequent test.

\section{EXPERIMENT 3}

The above analysis of outcome devaluation by specific satiety assumes that three features of this treatment interact to bring about a reduction in incentive value. First, the induction of a state of satiety results in the assignment of a low incentive value to any food or fluid experienced while in that state. Secondly, the low incentive value is associated with the sensory properties of the food or fluid, most notably its taste. Finally, we argue that this assignment comes under the conditional control of the specific state induced by the prefeeding. Experiment 3 was formulated to test whether the reduced value of an outcome induced by a specific satiety treatment could become conditional upon a highly specific sated state, as this account suggests.

According to the accounts of Experiments 1 and 2 presented above, it should be possible to devalue one outcome by a brief exposure to it immediately following consumption of another food to satiety. To assess this prediction, rats in Experiment 3 were trained in a fashion similar to that used in Experiments 1 and 2, except that, in this experiment, chainpulling replaced leverpressing as one of the two instrumental actions and, instead of polycose, a very similar polysaccharide, maltodextrin, was used, such that one action delivered salt maltodextrin and performance of the other delivered sour maltodextrin. After this training, all rats were exposed to two specific-satiety relationships. In one session, the rats were allowed to consume one type of flavored food pellet to satiety for $1 \mathrm{~h}$ prior to being given a brief, 10 -min exposure to one of the two maltodextrin solutions used in training. In the second consumption session, the rats consumed another differently flavored food pellet for $1 \mathrm{~h}$ prior to a brief exposure to the second maltodextrin outcome. According to the incentive learning analysis presented above, this specific-satiety treatment should lead to the devaluation of one of the outcomes conditional upon the state of satiety induced by consuming one type of food pellet, whereas devaluation of the other outcome should be conditional upon the state of satiety induced by consuming the other type of food pellet. To assess this prediction, the rats were given a choice between the two instrumental actions in an extinction test following prefeeding on one type of pellets. Prefeeding with one type of pellet should produce a selective devaluation of the outcome that was associated with those pellets during the specific-satiety treatment, and so, from the incentive learning account presented above, it may be predicted that the performance of the action trained with this outcome should be reduced relative to the other action in the extinction test.

\section{Method}

Subjects and Apparatus. Sixteen naive adult male hooded Lister rats were housed in squads of 4 and maintained under conditions similar to those described in Experiment 1. The four Campden Instruments operant chambers (Loughborough, England) used for training and testıng had a configuration similar to those employed in the previous studies, except that, instead of the syringe pumps, each operant chamber was fitted with a peristaltic pump that delivered a solution of $20 \%$ maltodextrin (Cerestar, Manchester, England) dissolved in $0.1 \mathrm{M}$ sodium chloride into the recessed magazine and a dipper that could deliver $20 \%$ maltodextrin containing $3 \%$ lemon juice. Each outcome presentation consisted of the delivery of $0.05 \mathrm{ml}$ of the appropriate solution. In addition, the left-hand lever in each chamber was replaced by a chain suspended from a microswitch through the celling so that it hung opposite the aperture for the lever, which was blanked off, $3.5 \mathrm{~cm}$ from the front wall. Each chamber was iluminated by a $3-W, 24-V$ houselight mounted in the center of the front wall above the flap door that gave access to the magazine. A BBC microcomputer (Acorn, Cambridge, England), equipped with the SPIDER extension for on-lıne control (Paul Fray, Cambridge, England), controlled the equipment and recorded the leverpresses and chainpulls. During the specific-satiety phase (see below), two types of 45-mg Noyes pellets were used: formula $A$ was presented in one session and formula $P$ in the other session, counterbalanced across rats. Because the rats were group housed in this study, instead of using the home cage, specific-satiety training was conducted in plastic feeding cages $(30 \mathrm{~cm}$ long $\times 13 \mathrm{~cm}$ wide $\times$ $11 \mathrm{~cm}$ high) with wire-mesh ceilings, each of which contained either a small glass food dish or a graduated plastic drunking tube. When glass dishes were presented, they always held $50 \mathrm{~g}$ of the appropriate pellets, and when drinking tubes were presented, they always held $50 \mathrm{ml}$ of the appropriate solution.

Procedure. The procedure for instrumental training was similar to that described in Experiment 1 . The rats were maintained on a 22.5-h food deprivation schedule throughout instrumental training. After two sessions of magazine training, all animals were first trained to chainpull in the absence of the lever and then, in the subsequent session, to leverpress in the absence of the chain. For 8 of the subjects, pressing the lever delivered the sour maltodextrin outcome and pulling the chain delivered the salt maltodextrin outcome; the remaining subjects received the opposite action outcome assignment. During concurrent training with both the lever and the chain, the outcomes became available on the single RI schedule, as described in Experiment 1. The nominal RI schedule value was 2 and $7 \mathrm{sec}$ for the first two sessions of concurrent training and $15 \mathrm{sec}$ for the next four sessions. The start of each session was signaled by the onset of the houselight and terminated by 1 ts offset. For 15 min following each of the final two training sessions, the animals were placed in the feeding cages to allow habituation to that environment prior to the consumption phase.

On the day after the final instrumental training session, all anımals were placed in the feeding cages and given the opportunity to 
consume one type of food pellet, either formula $A$ or formula $P$, from the glass dishes for $1 \mathrm{~h}$. For half of the anımals, the pellets were formula $\mathrm{A}$, and for the remainder, formula $\mathrm{P}$, counterbalanced with respect to the action-outcome assignments. Immediately after this period, the glass dishes were removed. Half of the animals exposed to each pellet type then received $10-\mathrm{min}$ access to the sour maltodextrin outcome presented in the graduated drinkıng tubes, with the remainder receiving the salt maltodextrin outcome. At least $2 \mathrm{~h}$ after this session, all animals were allowed 30 -min access to their maintenance diet. The next day, all animals were placed in the feeding cages again and were allowed to consume the other type of food pellet for $1 \mathrm{~h}$. Thus, if the first exposure on the previous day had been to formula A, on this day it was to formula P. After this first period, animals were given 10-min exposure to the other instrumental outcome; for example, if in the previous session they had received sour maltodextrin, in this session they recerved the salt maltodextrin. Again, all animals were allowed 30-min access to their maintenance diet at least $2 \mathrm{~h}$ after this session.

On the day following the second specific-satiety session, all animals were replaced in the feeding cages and given one type of food pellet for $1 \mathrm{~h}$. For half of the animals, the prefed pellets were formula $A$; for the remainder, they were formula $P$. The animals were then returned to the operant chambers, and performance of the two actions was assessed in a single 12-min choice extinction test.

\section{Results and Discussion}

The two actions were distinguished in terms of whether they had been trained with the outcome that, during the specific-satiety training, had been presented following the type of food pellets prefed prior to the test. The revalued outcome was that paired with the prefed pellets, and the nonrevalued outcome was that paired with the other pellets. Inspection of performance during the choice extinction test revealed that the difference between the rates of the actions trained with the revalued and nonrevalued

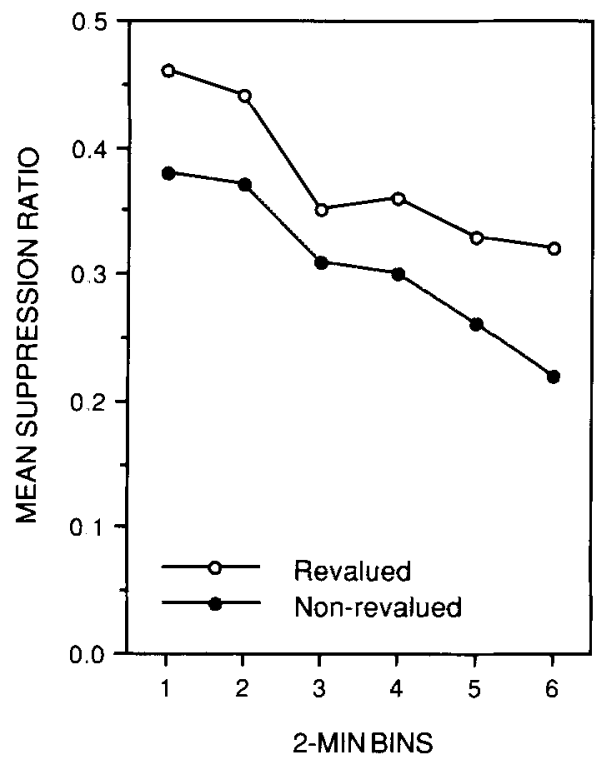

Figure 3. Experiment 3: Mean suppression ratios during the extinction test. The results are presented separately for the action that, in training, delivered the outcome that was subsequently revalued by the specific-satiety treatments and by prefeeding prior to the test and for the action that, in training, delivered the outcome that was not revalued by prefeeding prior to the test. outcomes failed to reach significance $[F(1,15)=2.97$, $p=.1]$. The mean rates of performance for the actions trained with the revalued and nonrevalued outcomes were, respectively, 4.5 and $3.7 / \mathrm{min}$. To minimize the variance introduced by differences in the rates at which the two actions were performed during training, we transformed the extinction test performance of each action in each 2-min bin using the ratio $A /(A+B)$, where $A=$ rate of test performance and $B=$ rate of performance in the final instrumental training session. The results of this transformation are presented in Figure 3, which shows that the animals performed the action trained with the revalued outcome relatively more than the action trained with the nonrevalued outcome $[F(1,15)=6.25]$, an effect that did not vary significantly across the test session $(F<1)$.

To establish that the interpretation of the ratios was not confounded by differences in baseline rates, an analysis was conducted on the data from the final training session. This analysis revealed that performance of the action whose outcome was to be revalued did not differ from performance of the action trained with the other outcome $(F<1)$. In the final training session, the two actions were performed at mean rates of 7.3 and $7.8 / \mathrm{min}$, respectively.

The revaluation effect observed in this study is exactly the opposite of the one predicted by the incentive learning analysis of outcome devaluation by specific satiety presented above. This analysis anticipates that an action trained with the revalued outcome should be performed less frequently than one associated with the nonrevalued outcome, because the revalued outcome, having been previously experienced in the specific sated state induced by prefeeding on one type of pellets, should have a lower incentive value when that state is reinstated by prefeeding on test. In contrast to this prediction, however, the revalued action was performed significantly more frequently than the nonrevalued one.

Although this finding challenges the incentive learning account of outcome revaluation by specific satiety that motivated this study, it does, nevertheless, demonstrate that the incentive value of an outcome can be controlled by a specific state of satiety. In the absence of such control, it is not clear how prefeeding with a particular type of food pellet could selectively enhance performance of the action trained with the outcome associated with satiety for those pellets. Rather than controlling a low-incentive value, however, the satiety state induced by prefeeding appears to enhance the value of the associated outcome.

In retrospect, there are grounds for anticipating just such an enhancement. The standard specific-satiety effect in consumption shows that, having reduced intake by satiety on one food, consumption can be reinstated by switching to a novel food (see Rolls, 1990, for a review). Thus, it is plausible that a varied incentive learning treatment that involves the introduction of a different food immediately after being sated on another type enhances, rather than reduces, the incentive value of the second food by a form of contrast process, and that this enhancement is controlled by the specific state of satiety induced by con- 
sumption of the first food. The next studies examined a further prediction of this modified incentive learning account of outcome revaluation by specific satiety.

\section{EXPERIMENTS 4A AND 4B}

The modified incentive learning account applied to the results of Experiment 3 predicts that the varied specific-satiety treatment employed in Experiment 3 should induce a more substantial outcome revaluation effect than is usually observed following specific-satiety-induced devaluation. This prediction follows from the fact that the varied treatment, in which the animals are allowed to consume one food to satiety before receiving a relatively brief exposure to a second food, engages two sources of revaluation. The first is the standard devaluation of the sated food, whereas the second is the inflation of the incentive value of the subsequently contacted food outcome that was apparent in Experiment 3 . Thus, if the first food is employed as the outcome of one instrumental action and the second food as the outcome of another action, an enhanced revaluation effect should be observed when the animals are tested for the performance of the two actions following prefeeding of the first outcome.

Experiments $4 \mathrm{~A}$ and $4 \mathrm{~B}$ were designed to test this prediction. Rats were trained, as in previous studies, on two actions, with one action trained with one outcome and the other action with a second outcome. During the specificsatiety phase, one group, Group Varied, received two varied-consumption sessions similar in procedure to those conducted in Experiment 3. In the first consumption session, the rats were allowed to consume one of the two instrumental outcomes for $1 \mathrm{~h}$, followed by a relatively brief, 10-min exposure to the other instrumental outcome. In the second session, the roles of the outcomes were reversed. As a consequence of this treatment, the modified incentive learning account predicts that subsequent extended exposure to one of the two outcomes should not only devalue the prefed outcome but should also elevate the incentive value of the other outcome. Thus, an instrumental choice extinction test should reveal an enhanced revaluation effect following extended prefeeding with one of the two outcomes. In Experiments 4A and 4B, this enhancement was assessed by contrasting the test performance of Group Varied with that shown by a second group, Group Consistent, in which only the usual devaluation process should operate. For these animals, each session of the specific-satiety treatment consisted of $1-\mathrm{h}$ exposure to one of the outcomes, followed by a further 10-min presentation of the same outcome, with one outcome presented in this manner in the first session and the other in the second session. This consistent specificsatiety treatment should result only in the assignment of a low incentive value to the prefed outcome on test without any elevation in the value of the other outcome. Thus, in summary, the modified incentive learning account pre- dicts that a larger revaluation effect should be observed in Group Varied than in Group Consistent, with the difference being primarily due to an elevation in the performance of the action trained with the nonprefed outcome.

This prediction was examined in two replications that differed primarily in the foods employed as the two outcomes. The first replication (Experiment 4A) used highly discriminable outcomes, food pellets and the sour maltodextrin solution, which differ in a number of nutritional and sensory features. By contrast, to establish whether any increment in the devaluation effect induced by the varied treatment, like the devaluation effects observed in the first two studies, could be mediated by taste alone, the outcomes in the second replication (Experiment 4B) were the salty and sour polycose solutions.

\section{Method}

Subjects and Apparatus. Experıment 4A employed 16 nave adult male hooded Lister rats, which were housed and maintained as in Experiment 3. Training and testing took place in the same operant chambers used in Experiment 3, except for the replacement of the pump by a dispenser that delivered 45 -mg Noyes pellets (formula A) into the magazıne. Sixteen female Long-Evans rats were used in Experıment 4b. These animals were housed and maintained under the same conditions and were trained in the same apparatus with the same outcomes as those employed in Experiment 1.

Procedure. The instrumental training procedures for Experiments $4 \mathrm{~A}$ and $4 \mathrm{~B}$ were the same as those employed in Experiments 3 and 1 , respectively. After two sessions of magazine trainıng, all anımals were first trained to perform one action and then, in the subsequent session, to perform the other on a continuous reinforcement schedule for different outcomes. During concurrent trainıng, the outcomes became avallable on a single RI schedule with a parameter value that was progressively increased across the first three sessions of concurrent training to $15 \mathrm{sec}$, at which it remained for a further four sessions. The sessions terminated after 15 of each of the two outcomes had been delivered. Leverpressing and chainpulling were trained with the food pellets and the sour maltodextrin solution as outcomes in Experiment 4A; left and right leverpressing were trained with the salty and sour polycose solutions as outcomes in Experiment 4B. The action- outcomes assignments were counterbalanced within each study.

After the final session of instrumental training, half of the animals in each training condition were assigned to one of two groups $(n=8)$ for the specific-satiety treatment -Group Consistent and Group Varıed. On the day after the final training session, each an1mal was given the opportunity to consume one of the outcomes for $1 \mathrm{~h}$. Immediately after that period, the drinking bottles or food bowls were removed, and Group Consistent received a further 10-min exposure to the same outcome; Group Varied also received a further 10 -min exposure but to the other outcome. At least $2 \mathrm{~h}$ after this session, all animals were allowed 30 -min access to their maintenance diet. The next day, all animals were allowed first to consume for $1 \mathrm{~h}$ the outcome that they did not consume first the previous day. Thus, for example, if the first exposure on the previous day had been the sour maltodextrin (Experiment 4A) or the sour polycose (Exper1ment $4 B$ ), then, on this day, it was, respectively, to the food pellets or to the salt polycose. Again, animals in Group Consistent were given a further 10-min exposure to the same outcome at the end of that hour, whereas anımals in Group Varied received the other outcome for this period, with all animals receiving 30-min access to their maintenance diet at least $2 \mathrm{~h}$ after this session. 


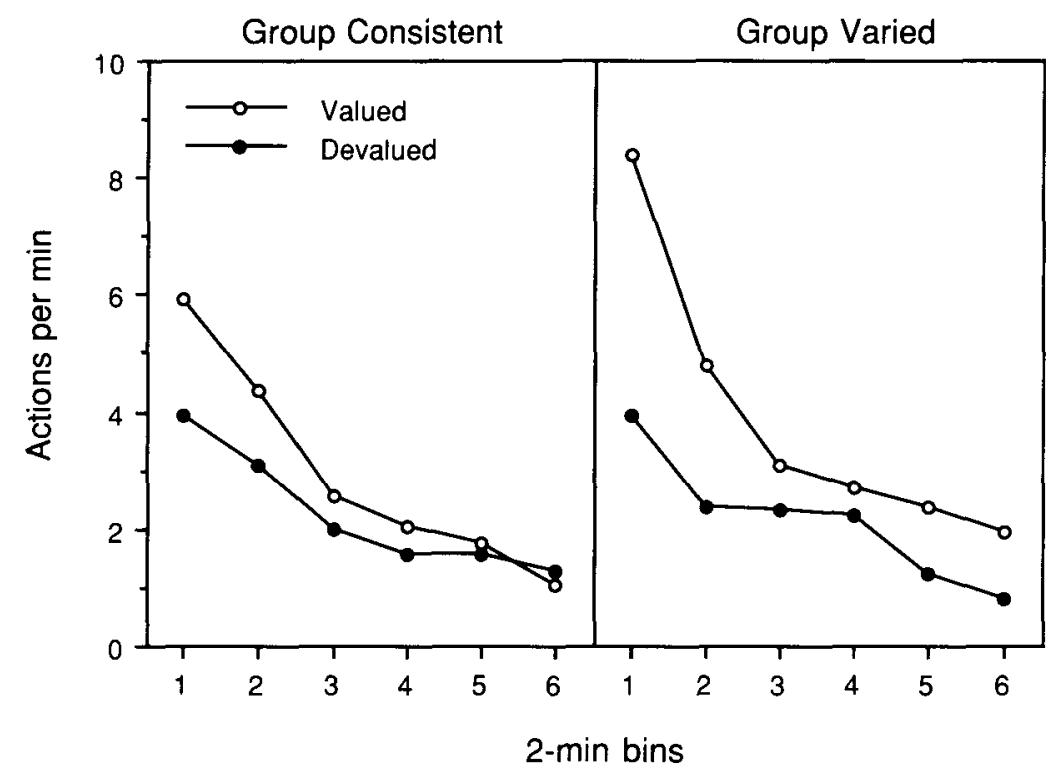

Figure 4. Experiment 4A. Mean leverpresses and chainpulls per minute during the choice extinction tests. The results are separated by group: Group Consistent (left panel) was exposed to the same outcomes during both periods of each specific-satiety treatment session; Group Varied (right panel) was exposed to different outcomes during the two periods of each specific-satiety treatment session. The results for each group are presented separately for the action that, in training, delivered the outcome subsequently devalued by the specific-satiety treatment and by prefeeding prior to the test, and for the action that, in training, delivered the outcome that remained valued.

On the following day, all animals were presented with one of the two instrumental outcomes for $1 \mathrm{~h}$. The prefed outcome was counterbalanced with respect to the action-outcome assignments within each group. Immediately after this prefeeding period, the animals were placed in the operant chambers and performance was assessed in a single choice extinction test of $12-$ min duration and on the levers and chains in Experıment 4A, but of only 10-min and on the two levers in Experiment 4B. No outcomes were delivered during this session.

\section{Results and Discussion}

Performance during the extinction tests of Experiments $4 \mathrm{~A}$ and $4 \mathrm{~B}$ are presented in Figures 4 and 5, respectively, separately for the consistent (left panel) and varied groups (right panel). The devalued action refers to the action trained with the outcome to which animals were exposed immediately prior to the test and the valued action to the action trained with the other outcome. The test performance of the consistent groups in both experiments replicated the devaluation effect, observed in previous experiments, induced by the specific-satiety treatment. The animals performed the action trained with the devalued outcome less than they did the other action. More importantly, however, the magnitude of this revaluation effect was larger in Group Varied than in Group Consistent in both studies. Moreover, this enhancement appeared to be due to an elevation in the performance of the action trained with the valued outcome.

To assess this description of the data, we conducted three-way mixed analyses of variance using a between- subjects factor of group and a within-subjects factor of revaluation, which distinguished the performance of the action whose training outcome was prefed immediately prior to the test from the other action, and of bin, which separated performance in each of the 2-min bins in each test. In reporting results of these analyses, the $F$ ratios for Experiment $4 \mathrm{~A}$ will be presented first, followed by those for Experiment 4B. These analyses revealed significant overall effects of revaluation $[F(1,14)=19.5$ and $53.8]$ and, importantly, significant group $\times$ revaluation interactions $[F(1,14)=7.5$ and 14.0$]$ and significant threeway interactions $[F(5,70)=2.1$ and $F(4,56)=3.9]$, offering consistent support for the suggestion that the effect of revaluation was larger in Group Varied than in Group Consistent in both studies. Simple main effects analyses, conducted to assess the source of the significant group $\times$ revaluation interactions, revealed reliable differences between groups in their performance of the action that, in training, had delivered the outcome that was valued $[F(1,14)=4.9$ and 5.4$]$, whereas there were no such differences between groups in their performance of the action that, in training, had delivered the outcome that was devalued $(F \mathrm{~s}<1)$.

These effects found in the extinction test were not present during training. Analysis of performance during the final concurrent training session revealed no effects of group or revaluation and no interaction between these factors (all $F \mathrm{~s}<1$ ). The mean number of actions per minute that, in training, delivered the outcome that was devalued 


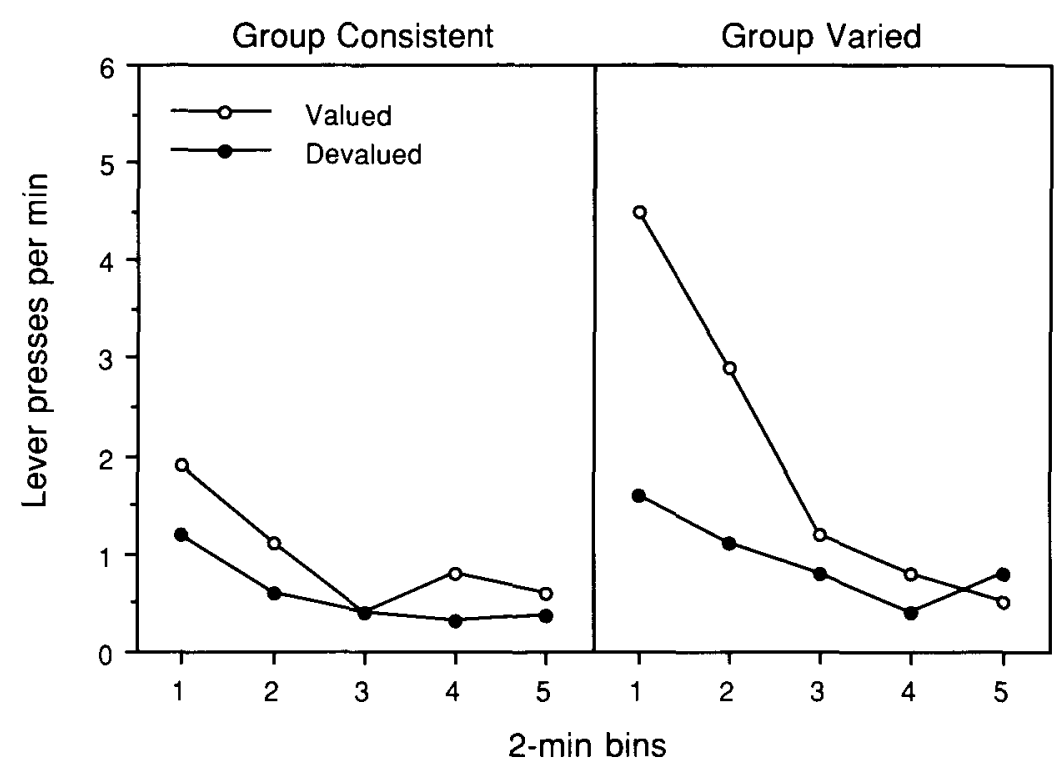

Figure 5. Experiment 4B: Mean leverpresses per minute during the choice extinction tests. The results are separated by group: Group Consistent (left panel) was exposed to the same outcomes during both periods of each specific-satiety treatment session; Group Varied (right panel) was exposed to different outcomes during the two periods of each specific-satiety treatment session. The results for each group are presented separately for the action that, in training, delivered the outcome subsequently devalued by a specific-satiety treatment and prefeeding prior to the test, and for the action that, in training, delivered the outcome that remained valued.

and valued, respectively, prior to the test were: for Group Consistent, Experiment 4A, 7.7 and 7.6, and Experiment 4B, 6.1 and 5.9; for Group Varied, Experiment 4a, 7.7 and 7.5, and Experiment $4 \mathrm{~b}, 6.0$ and 5.9.

These results are exactly those anticipated if the specific-satiety treatment acted both to devalue the prefed outcome and, in Group Varied, to induce a relative increment in the incentive value of the other instrumental outcome. Thus, we observed enhanced revaluation effects in varied groups which were due to the fact that animals in these groups performed the action trained with the nonprefed outcome more than did the animals in consistent groups. The specific-satiety regime of the varied condition acted to enhance the incentive value of the outcome that was experienced immediately after feeding to satiety on another outcome. Moreover, the enhancement must have been under the conditional control of the satiety state induced by the prefeeding as the specific-satiety treatment was identical for the two outcomes. Importantly, the fact that the augmented revaluation effect was observed in Group Varied of Experiment 4B shows that, like the devaluation evident in Group Consistent, the enhancement of incentive value can be mediated solely by the taste of an outcome.

As we have already noted, the enhancement of incentive value produced by the varied regime may be related to the specific-satiety effect observed in food consumption in both rats (e.g., Le Magnen, 1967) and human subjects (Rolls, 1990). This effect was also observed during the specific-satiety phases of the present experiments.
Figure 6 illustrates consumption during the first 1 -h period and during the second $10-\mathrm{min}$ period for the two groups in Experiment 4A, separately for the food pellets (left panel) and the maltodextrin solution (right panel). Although the consumption was comparable in the first periods, all the animals in Group Varied ate more pellets and drank more maltodextrin in the second period than those in the Group Consistent. Figure 7 shows that a similar specific-satiety effect was observed in the consumption phase of Experiment 4B. Although it is clear, from this figure, that the renewed consumption in Group Varied of Experiment 4B was not as dramatic as that found in Experiment 4A, it is important to note that all of the animals in Group Varied of Experiment 4B drank more polycose during the second period when it had a different taste from that presented in the first period than did Group Consistent, for which the taste was the same in the two periods.

\section{GENERAL DISCUSSION}

In general, the present experiments have established a role for incentive learning in the revaluation of an instrumental outcome by a specific-satiety treatment. More specifically, they suggest that when animals are sated on a particular food commodity, they learn that the taste of that food has a relatively low incentive value and can also learn that subsequently contacted foods that differ in taste have a relatively high incentive value. Furthermore, it is clear that, given sufficient opportunity, the state of 


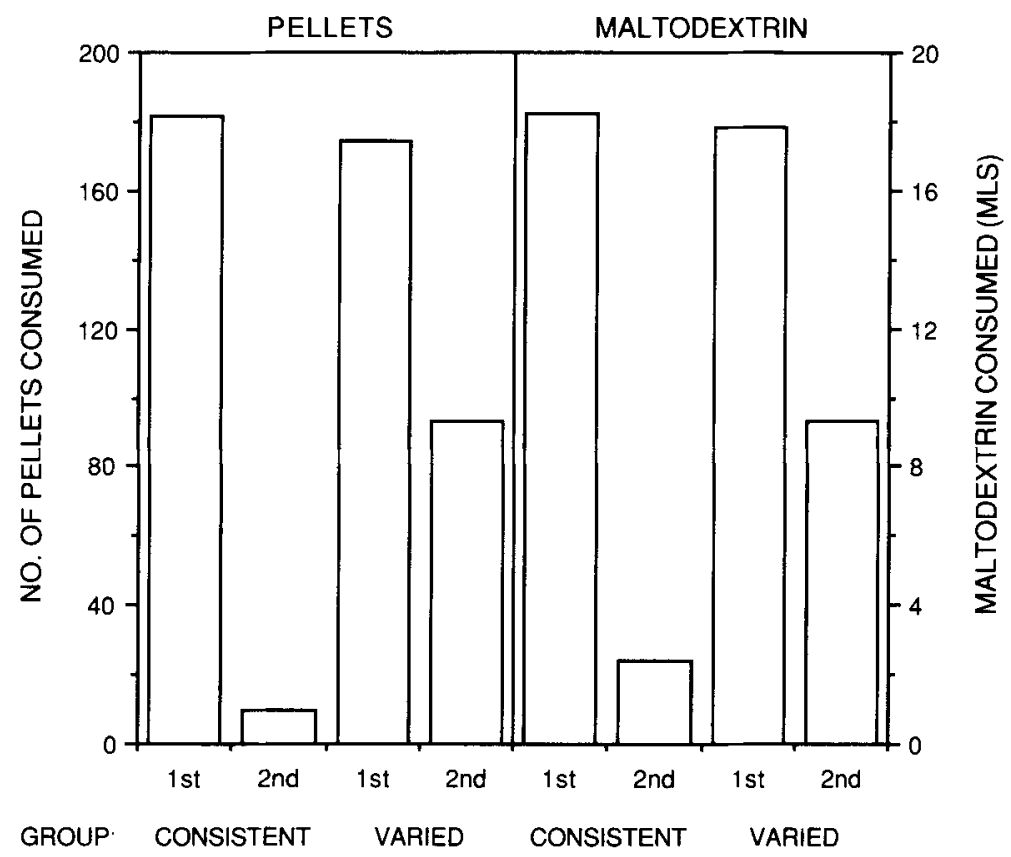

Figure 6, Experiment 4A: Mean number of pellets (left panel) and volume of maltodextrin solution (right panel) consumed in first and second periods of the specificsatiety treatment sessions by the consistent and varied groups.

satiety induced by extended exposure to a particular outcome can acquire conditional cuing properties that can signal both the reduced incentive value of the food on which a rat is sated and an increase in the value of subsequently contacted, different tasting foods. These conclusions are based on a number of findings.

As has been reported for devaluation by conditioned taste aversion (Balleine \& Dickinson, 1991; Rescorla, 1990), Experiment 1 demonstrated that outcome devaluation by specific satiety can be mediated by a change in the incentive value of specific taste features of the instrumental outcome. When rats were trained to perform two leverpress actions, one delivering salty tasting polycose and the other sour tasting polycose, sating them on one of these two polycose solutions prior to a choice extinction test on the levers induced a selective reduction in performance on the lever that had delivered, in training, the polycose solution on which they were sated.

On the basis of an analysis of previous demonstrations of devaluation induced by a posttraining shift in primary motivation, we argued that this specific-satiety induced reduction in incentive value may have been mediated by incentive learning. We assessed this hypothesis in Experiment 2 by attempting to separate the opportunity for learning about the change in the value of specific taste features following satiety from the satiety treatment applied prior to the choice extinction test. To achieve this, hungry rats were trained as in Experiment 1 and were then, in an incentive learning phase, given a 1-h exposure to either the sour or the salt polycose solution on each of the next 2 days. Following this treatment, rats were given two choice extinction tests on the levers: one was conducted following 1-h exposure to an unadulterated polycose solution and the other was conducted in the absence of this exposure, that is, when the rats were hungry. Ev-

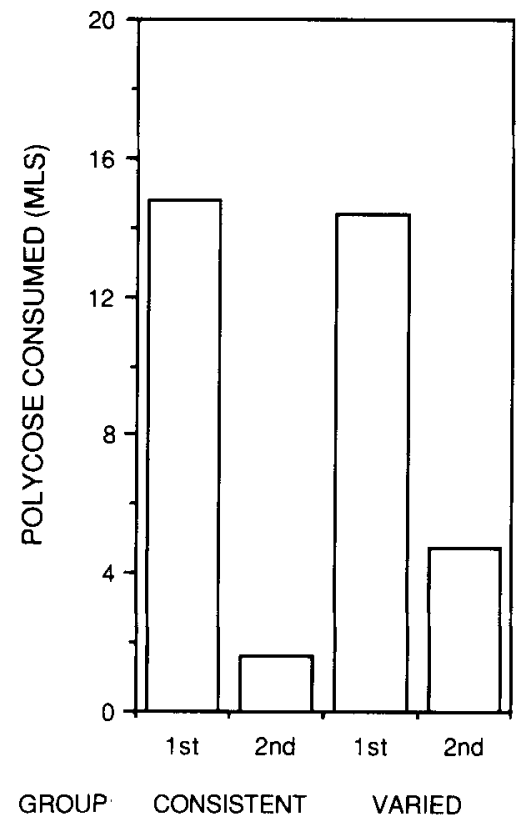

Figure 7. Experiment 4B: Mean amount of the polycose solutions consumed during the first and second periods of the specific-satiety treatment sessions by Group Consistent (left two columns) and Group Varied (right two columns). 
idence that specific-satiety induced devaluation depends on incentive learning was derived from the finding that, although performance did not differ in the test conducted hungry, after rats were sated on the unadulterated polycose they performed fewer incidents of the action that, in training, had delivered the polycose solution on which they were sated during the incentive learning phase than they did the other action.

One interpretation of this result is that sating hungry rats on a polycose solution containing a particular taste allows them to learn that, with increasing satiety, the taste has a reduced incentive value relative to when it is consumed hungry. Furthermore, this reduction in value must clearly be able to be brought under the conditional control of the state of satiety induced by consumption of the accompanying polycose, because the devaluation effect emerged in the test conducted after satiety on the polycose but not in the test conducted hungry. It may, however, be argued that this result reflects the operation of within-compound associations. If it is assumed that the incentive learning treatment used in Experiment 2 acted differentially to strengthen a particular polycosetaste association, then a ready account can be given of these results on the basis of the devaluation of an associate without reference to incentive learning and the conditional control by satiety.

The results of Experiments 3 and 4 do not support the latter account, however. In the incentive learning phase of Experiment 3, one or the other variety of Noyes pellets was consumed before brief exposure to one or the other of the two polycose solutions. Although this should clearly have allowed the formation of relatively specific within-compound associations, sating rats on one of the two types of pellets prior to the choice extinction test did not produce a conditional devaluation effect. Rather, the rats increased their performance of the action that, in training, had delivered the polycose solution exposed after consumption of that kind of pellet. Furthermore, the account of Experiment 2 developed using withincompound associations cannot predict the results found in Experiment 4B. Group Consistent and Group Varied in the latter experiment were given the same exposure to both polycose solutions during training and incentive learning phases, and yet the devaluation effect that emerged after prefeeding on one polycose solution was far greater in Group Varied than in Group Consistent. As a consequence, we favor the incentive learning account of the results of Experiment 2.

It is of some interest that Experiment 3 demonstrated that specific-satiety processes could produce a relative enhancement of incentive value. Brief exposure to an instrumental outcome after extended exposure to the one type of food pellet produced a conditional inflation of incentive value rather than a devaluation. This finding was interpreted as evidence that specific-satiety revaluation treatments could produce two effects on outcome value. First, satiety acts both to reduce the incentive value of nutrient outcomes and to induce an internal state upon which that change in value can become conditional. Secondly, because the induction of satiety can cause a reduction in the incentive value of a nutritive outcome that is relatively specific to its taste features, the incentive value of nutritive outcomes with different taste features can remain at least relatively unaffected, thereby allowing a form of incentive contrast between sequentially presented outcomes to develop (Flaherty, 1982). This contrast could serve to elevate the incentive value assigned to the second outcome relative to the first. As presented in the introduction to Experiment 4, this type of twoprocess account predicts that a specific-satiety treatment in which rats experience a second instrumental outcome following the induction of satiety on a first should produce a larger devaluation effect than a treatment consisting solely of the induction of satiety with a single outcome. This is because the latter procedure serves to devalue only one outcome (conditional on the state induced by prefeeding this outcome), whereas the former, varied, procedure also inflates the incentive value of the second outcome. This prediction was confirmed in the final two studies, which also demonstrated, importantly, that the augmented revaluation effect could be mediated solely by the taste of the outcome.

This conclusion has important implications for the effects of incentive learning on instrumental performance following a posttraining shift in primary motivation (e.g., Balleine, 1992; Balleine \& Dickinson, 1994; Balleine, Garner, Gonzalez, \& Dickinson, 1995). In these studies, rats are trained when hungry to perform two instrumental actions for different nutritive outcomes. Prior to a choice extinction test, the animals are sated on their maintenance diet and then allowed brief contact with one of the two instrumental outcomes while in this sated state; the extinction test is also conducted with the rats in the sated state. Although we have consistently found that this treatment is sufficient to reduce the performance of the action that, in training, delivered the outcome exposed when satiated, careful consideration of the results of Experiments $3,4 \mathrm{~A}$, and $4 \mathrm{~B}$ of the current study suggests that this effect may have been reduced by the type of incentive contrast effect found in these experiments. Thus, although exposure to the instrumental outcome when satiated allows animals to learn about the reduced value of the outcome when in that state, it is clear that the value of the taste of this nutritive outcome must be greater than the taste of the animals' maintenance diet, which they have been allowed to consume to satiety. Thus, the state of satiety may signal the reduction in the value of the outcome relative to its value in training and, hence, relative to the other, nonreexposed, outcome, but the sensory properties of the maintenance diet may also signal that the reexposed outcome has at least some positive incentive value. Thus, responding on test may not reflect the full effect of satiety. This account predicts that if animals were sated by a means that does not require consummatory contact (and hence exposure to the taste features of the maintenance diet), for example, by means of intubation, a greater ef- 
fect of incentive learning may be found, a prediction that awaits a definitive test.

It remains to be considered how specific-satiety treatments act to cause changes in the incentive value of the instrumental outcome. As we have already noted, the inflation of incentive value observed in Experiments 3, 4A, and $4 \mathrm{~B}$ is consistent with a number of previous assessments of specific-satiety effects in food consumption by humans and animals (see Rolls, 1990, for a review). Indeed, a consistent increase in consumption was observed in Experiments 4A and 4B when the subsequently exposed outcome differed in taste from that previously consumed. The fact that a switch to a novel food enhances its hedonic impact in humans (e.g., Hetherington, 1996; Rolls, Hetherington, \& Burley, 1988; Rolls, Laster, \& Summerfelt, 1989; Rolls, Rolls, Rowe, \& Sweeney, 1981; Wooley, Wooley, \& Dunham, 1972) suggests that the increment in consumption and in incentive value is based on a relative enhancement of the affective response to the newly consumed food. This position contrasts with that of Cabanac (1971), who has argued that the reinitiation of feeding in these cases reflects the increased "usefulness" of the novel food relative to that on which the humans or animals were initially sated.

Although Cabanac's (1971) position has been influential, there is considerable evidence, as with other means of devaluing the instrumental outcome, that specific-satiety effects in both humans and rats are mediated by changes in palatability processes rather than by signals related to the "usefulness" or nutritional consequences of consumption. Thus, for example, for human subjects, Cabanac reported that prior consumption of glucose reduced the rated hedonic impact of $20 \%$ sucrose and concluded that the calories in glucose reduced the usefulness of the sucrose, whereas Wooley et al. (1972) found in a subsequent study that the nonnutritive sweetener cyclamate was just as effective at reducing the hedonic impact of the sucrose. Furthermore, Rolls et al. (1988) reported that macronutrient structure was not a sufficient condition for the generalization of specific satiety between different commodities and that such transfer was predominantly based on taste. Finally, changes in the rated hedonic impact that accompany a specific-satiety treatment in humans can be very rapid. For example, in studies conducted by Rolls et al. (1989) and Hetherington, Rolls, and Burley (1989), a change in hedonic rating was sometimes found within 2 min of consumption, a change that is difficult to account for in terms of changes in "usefulness."

In rats, the evidence is more equivocal, largely due to the difficulties involved in assessing changes in the hedonic impact of a commodity following a specificsatiety treatment. Nevertheless, Le Magnen's (1967) early finding that the food consumption of rats is increased by over $70 \%$ when they are given a varied diet points to the fact that the consumption of new foods is not based solely on "usefulness." An indirect means developed to assess the affective response of rats to foods and fluids is that based on taste reactivity, reflected in changes in the fixed action patterns that accompany the ingestion of palatable commodities (Grill \& Norgren, 1978). Using this measure, Berridge (1991) found that reactions that accompany ingestion were reduced more to a nutritive solution on which rats were sated than to an equicaloric solution on which they were not sated. Finally, in studies of instrumental outcome devaluation in rats, there is evidence that changes in the incentive value of the instrumental outcome are accomplished rapidly. Dickinson and Balleine (1995) report one study in which a rapid effect of a shift from hunger to satiety on instrumental performance for a food pellet outcome was found. The instrumental performance of sated animals with no previous experience with the food pellet outcome when in that state declined to the level of those with such prior experience after the receipt of, on average, only two pellets.

Whatever the mechanisms by which specific satiety treatments induce modifications in the incentive value of an instrumental outcome, the current results suggest that, as with a number of other examples of instrumental outcome revaluation, revaluation by specific satiety can involve an incentive learning process. There is now evidence to suggest that incentive learning modifies the effectiveness of outcome revaluation treatments when outcomes relevant to hunger (Balleine, 1992; Balleine \& Dickinson, 1994), thirst (Lopez, Balleine, \& Dickinson, 1992), thermoregulatory (Hendersen \& Graham, 1979), and sexual needs (Everitt \& Stacey, 1987) are revalued by a shift in the relevant motivational state, when outcomes are revalued by drug treatments (Balleine, Ball, \& Dickinson, 1994), and when outcomes are devalued by conditioning a taste aversion to them (Balleine \& Dickinson, 1991, 1992; Balleine, Garner, \& Dickinson, 1995). The current experiments serve to extend our knowledge of the generality of incentive learning to another means of revaluing the instrumental outcome; that induced by outcome-specific satiety.

\section{REFERENCES}

Adams, C. D., \& Dickinson, A. (1981). Instrumental responding following reinforcer devaluation. Quarterly Journal of Experimental Psychology, 33B, 109-122

Balleine, B. W. (1992). The role of incentive learning in instrumental performance following shifts in primary motivation. Journal of Experimental Psychology Animal Behavior Processes, 18, 236-250.

Balleine, B. W, Ball, J., \& Dickinson, A. (1994). Benzodiazepineinduced outcome revaluation and the motivational control of instrumental action. Behavioral Neuroscience, 108, 573-589

BALleiNe, B. W., \& Dickinson, A. (1991). Instrumental performance following reinforcer devaluation depends upon incentive learning. Quarterly Journal of Experimental Psychology, 43B, 279-296.

Balleine, B. W., \& Dickinson, A (1992). Signalling and incentive processes in instrumental reinforcer devaluation. Quarterly Journal of Experimental Psychology, 45B, 285-301

Balleine, B. W., \& Dickinson, A. (1994). The role of cholecystokinin in the motivational control of instrumental action. Behavioral Veuroscience, 108, 590-605.

Balleine, B. W., Garner, C., \& Dickinson, A. (1995). Instrumental outcome-devaluation is attenuated by the anti-emetic ondansetron Quarterly Journal of Experimental Psychology, 48B, 235-251.

Balleine, B. W, Garner, C., GonZ.Alez, F., \& Dickinson, A. (1995) 
Motivational control of heterogeneous instrumental chains. Journal of Experimental Psychology Animal Behavior Processes, 21, 203-217.

BERRIDGE, K. C. (1991). Modulation of taste affect by hunger, caloric satiety, and sensory-specific satiety in the rat. Appetite, 16, 103-120.

Cabanac, M. (1971). Physiological role of pleasure. Science, 173, 1103-1107

Colwill, R. C., \& Rescorla, R. A. (1985). Postconditioning devaluation of a reinforcer affects instrumental responding. Journal of $E x$ perimental Psychology Animal Behavior Processes, 11, 120-132.

Colwill, R. C., \& Rescorla, R. A. (1986). Associative structures in instrumental learning. In G, H. Bower (Ed.), The psychology of learning and motivation (Vol. 20, pp. 55-104). New York. Academic Press.

Dickinson, A., \& Balleine, B. [W.] (1994). Motivational control of goal-directed action. Animal Learning \& Behavior, 22, 1-18.

Dickinson, A., \& Balleine, B. W. (1995). The motivational control of instrumental action. Current Directions in Psychological Science, 4, 162-167.

Dickinson, A., Campos, J., Varga, Z. I., \& Balleine, B. W. (1996). B1directional instrumental conditioning. Quarterly Journal of Experimental Psychology, 49B, 289-306.

EVERITT, B. J., \& STACEY, P (1987). Studies of instrumental behavior with sexual reinforcement in male rats (Rattus norvegicus): II. Effects of preoptic area lesions, castration and testosterone. Journal of Comparative Psychology, 101, 407-419.

FL.AHERTY, C. F. (1982). Incentive contrast. A review of behavioral changes following shifts in reward. Animal Learning \& Behavior, 10, 409-440.

Grill, H. J., \& Norgren, R. (1978). Chronic decerebrate rats demonstrate satiation but not bait shyness. Science, 201, 267-269.

Hendersen, R. W., \& Graham, J (1979). Avoidance of heat by rats: Effects of thermal context on the rapidity of extinction. Learning \& Motivation, 10, 351-363.
Hetherington, M. M. (1996). Sensory-specific satiety and its importance in meal termination. Neuroscience \& Biobehavioral Reviews, 20, 113-117.

Hetherington, M. M., Rolls, B. J., \& Burley, V. J. (1989). The time course of sensory-specific satiety. Appetite, 12, 57-68.

Le Magnen, J. (1967). Habits and food intake In C. F. Code (Ed.), Handbook of physiology (pp. 11-13). Washington, DC. American Physiological Society.

Lopez, M., Balleine, B. [W.], \& Dickinson, A. (1992), Incentive learning and the motivational control of instrumental performance by thirst. Animal Learning \& Behavior, 20, 322-328.

RESCORLA, R. A. (1990). Instrumental responses become associated with reinforcers that differ in one feature. Animal Learning \& Behavior, 18, 206-211.

RoL.s, B. J. (1990). The role of sensory-specific satiety in food intake and food selection. In E. D. Capaldi \& T. L. Powley (Eds.), Taste, experience and feeding (pp. 197-209). Washington, DC: American Psychological Association.

Rolls, B. J., Hetherington, M., \& Burley, V. J. (1988). The specificity of satiety: The influence of foods of different macronutrient content on the development of satiety. Physiology \& Behavior, 43, 145-153

Rolls, B. J., Laster, L. J., \& Summerfelt, A. (1989). Hunger and food intake after consumption of low-calorie foods. Appetite, 13, 115-127

Rolls, B. J., Rolls, E. T., Rowe, E. A., \& SweEnEy, K. (1981). Sensory specific sat1ety in man. Physiology \& Behavior, 27, 137-142.

Wooley, O. W., Wooley, S. C., \& Dunham, R. B. (1972). Calories and sweet taste: Effects on sucrose preference in the obese and nonobese. Physiology \& Behavior, 9, 765-768.

(Manuscript received March 25, 1997; revision accepted for publication June 30, 1997.) 